\title{
Epidurografia lombossacra com diferentes volumes de iohexol em felinos
}

\section{Lumbosacral epidurography with different volumes of iohexol in cats}

\author{
Syduane Morais Leite Ramos ${ }^{1 *}$; Pedro Isidro da Nóbrega Neto'; \\ Carlos Ticiano Coutinho Ramos 3 ; Paulo Vinícius Tertuliano Marinho4; \\ Maria Andrea Amorim Ferreira ${ }^{5}$; Sérgio Ricardo Araújo de Melo e Silva ${ }^{2}$
}

\section{Resumo}

Objetivou-se neste trabalho determinar a migração cranial de diferentes doses de iohexol, administradas pela via epidural lombossacra, analisando possíveis efeitos sobre algumas variáveis fisiológicas, em felinos. Foram utilizados 10 felinos sem raça definida, cinco machos e cinco fêmeas não prenhes, clinicamente sadios, com $1 \pm 0,5$ anos de idade e pesando $2,2 \pm 3,1 \mathrm{~kg}$. Os animais foram sedados com xilazina ( $1 \mathrm{mg} / \mathrm{kg}$ IM) e, $15 \mathrm{~min}$. após, foi administrado o iohexol na dose de $0,22 \mathrm{~mL} / \mathrm{kg}(\mathrm{G} 1)$ e de $0,3 \mathrm{~mL} / \mathrm{kg}(\mathrm{G} 2)$, pela via epidural. Foram analisados: a migração cranial do meio de contraste, a ataxia produzida, a frequência cardíaca ( $\mathrm{FC}$ ), a duração da onda $\mathrm{P}$, o intervalo $\mathrm{P}-\mathrm{R}$, o complexo QRS, o intervalo Q-T, a frequência respiratória $(f)$ e a temperatura corpórea $\left(\mathrm{T}^{\circ} \mathrm{C}\right)$. No $\mathrm{G} 1$ o meio de contraste migrou em média até a vértebra L2 $(10,2 \pm 1,7 \mathrm{~cm})$, valores menores que os obtidos no $\mathrm{G} 2$ (L1 e 12,1 $\pm 2,9 \mathrm{~cm})$. Não houve variação estatisticamente significativa em FC, duração da onda P, intervalo $\mathrm{P}-\mathrm{R}$, duração do complexo QRS, intervalo Q-T e $f$. A $\mathrm{T}^{\circ} \mathrm{C}$ diminuiu significativamente no G1 a partir do T40, sendo inferior à do $\mathrm{G} 2$ no T60. Concluiu-se que a dose de $0,3 \mathrm{~mL} / \mathrm{kg}$ é potencialmente a mais indicada para o bloqueio anestésico dos pedículos ovarianos de felinos, quando empregando anestésicos locais, e que o iohexol não altera os parâmetros fisiológicos.

Palavras-chave: Anestesiologia, meio de contraste, radiologia

\footnotetext{
Abstract

The objective of this work determinate the cranial migration of different levels of iohexol, administrated by the lumbosacral epidural anesthesia, analyzing the possible effects under some physiological variables, in felines. It was used 10 felines with no defined breed, five males and five females no pregnant and healthy, with $1 \pm 0,5$ years old and weighting $2,2 \pm 3,1 \mathrm{~kg}$. The animals were sedated with xylazine $(1 \mathrm{mg} / \mathrm{kg} \mathrm{IM})$ and 15 minutes after, it was administrated iohexol in the dose of $0,22 \mathrm{~mL} / \mathrm{kg}$ (G1) and of $0,3 \mathrm{~mL} / \mathrm{kg}(\mathrm{G} 2)$, by the epidural anesthesia. Were analyzed: the cranial migration of the

${ }^{1}$ Médica Veterinária Autônoma, Campina Grande, PB. E-mail: syduane@hotmail.com

${ }^{2}$ Profs. Drs., Unidade Acadêmica de Medicina Veterinária, Patos, PB. E-mail: pedroisidro@ymail.com; silva_sergioricardo@, yahoo.com.br

${ }_{3}^{3}$ Discente do Programa de pós-graduação em Ciência Animal, Instituto Nacional do Semiárido, Areia, PB. E-mail: ticianoramos@ hotmail.com

${ }^{4}$ Discente do Programa de pós-graduação em Ciência Animal, Universidade Estadual de Londrina, UEL, Londrina, PR. E-mail: paulo_veter@hotmail.com

${ }^{5}$ Discente do Programa de pós-graduação em Zootecnia, Universidade Federal de Campina Grande, UFCG, Patos, PB. E-mail: andreaveterinaria@hotmail.com

* Autor para correspondência
} 
contrast, the consequent ataxia, the cardiac frequency (CF), the P wave duration, the P-R gap, the QRS complex, the Q-T gap, the respiratory frequency (f), the body temperature $\left({ }^{\circ} \mathrm{C}\right)$. In the $\mathrm{G} 1$ the contrast did a migration until the L2 vertebra $(10,2 \pm 1,7 \mathrm{~cm})$, lower values than the obtained in the G2 (L1 and $12,1 \pm 2,9 \mathrm{~cm})$. There was no significant statistical variation for FC, the P wave duration, the P-R gap, the QRS complex duration, the Q-T gap and $f$. The $\mathrm{T}^{\circ} \mathrm{C}$ decreased significantly in the $\mathrm{G} 1$ from the $\mathrm{T} 40$, and less than the $\mathrm{G} 2$ at $\mathrm{T} 60$. It can be concluded that the dose of $0,3 \mathrm{~mL} / \mathrm{kg}$ is potentially the most indicated to promote the anesthetic block of the ovarian pedicles inthe felines, when using local anesthetics, and the iohexol do not modify the physiological parameters.

Key words: Anesthesiology, contrast, radiology

\section{Introdução}

A escolha da técnica anestésica a ser empregada depende do tipo, localização e duração da cirurgia proposta, do estado de higidez, espécie, idade, raça, tamanho e temperamento do paciente, do custo do(s) agente(s) anestésico(s) e da disponibilidade de equipamentos e fármacos anestésicos (BRUNSON, 1990).

Uma das técnicas muito utilizadas é a anestesia epidural, que consiste na administração de um fármaco no espaço epidural, com a finalidade de bloquear os impulsos nervosos dos nervos espinhais dentro do canal espinhal, ou, a partir da estimulação de receptores específicos, de produzir analgesia regional (SKARDA, 1996). Esta técnica anestésica é empregada tanto em pequenos comograndes animais.

Para Futema (2009b), em animais de pequeno porte o local de punção mais adequado é a lombossacra, com o paciente em decúbito lateral ou esternal e os membros pélvicos tracionados cranialmente, tomando-se como referência as asas do íleo e o processo espinhoso dorsal da sétima vértebra lombar, localizando-se o ponto de punção caudalmente a este último. O local de punção deve ser tricotomizado e preparado assepticamente e a agulha estéril deve ultrapassar a pele e subcutâneo, ligamentos supra-espinhoso, intervertebral e amarelo, crepitando ao passar por este último e chegando finalmente ao espaço epidural.

A epidurografia consiste na técnica radiográfica, de fácil aplicação, que envolve a injeção de um meio de contraste no espaço epidural lombossacra.
Trata-se de uma das modalidades de diagnóstico por imagem comumente utilizada para a avaliação de doenças dessa região em pequenos animais.

Nesta técnica, são empregados alguns meios de contraste, dentre eles o iohexol o qual pertence à segunda geração dos compostos hidrofílicos iodados não-iônicos. Possui osmolaridade baixa, é solúvel em água e é um dos mais empregados para procedimentos de mielografia (WOOD; FARROW; FAIRBURM, 1995). Comparado ao metrizamide, causa menos alterações adversas, com excelente qualidade radiográfica (LEWIS; HOSGOOD, 1992).

Em seres humanos, os estudos referentes à migração de substâncias dentro dos espaços epidural ou subaracnóideo são levados a cabo com a introdução de meios de contraste radiográficos nestes sítios, e posterior exame radiográfico (VAS et al., 2003). Estes estudos são geralmente realizados antes que anestésicos locais sejam empregados por estas vias, com a finalidade de aferir qual o volume mais indicado para produzir anestesia em determinada área corpórea.

Como possíveis reações adversas decorrentes do uso do iohexol em cães, Lewis e Hosgood (1992) citam apnéia, vômito, convulsão e agravamento de sinais neurológicos, inclusive com casos de óbito.

Da mesma forma, na literatura revisada, não foram encontrados relatos do uso do iohexol pela via epidural, em felinos, o que torna instigante a avaliação do seu emprego, por esta via, com o intuito de determinar, de forma menos invasiva qual a extensão da migração cranial do meio de contraste 
quando administrado em diferentes doses.

Objetivou-se com este trabalho determinar a extensão da migração cranial obtida com duas doses de contraste radiográfico, administradas pela via epidural lombossacra, bem como os efeitos desta administração sobre a frequência e ritmos cardíacos, a frequência respiratória e a temperatura corpórea em felinos.

\section{Material e Métodos}

\section{Animais}

Foram utilizados 10 felinos sem raça definida, cinco machos e cinco fêmeas não prenhes, clinicamente sadios, com $1 \pm 0,5$ anos de idade e pesando 2,2 $\pm 3,1 \mathrm{~kg}$. Os animais foram obtidos junto a proprietários usuários do Hospital Veterinário (HV) da Universidade Federal de Campina Grande (UFCG) - Campus de Patos, e foram trazidos ao HV apenas no dia do experimento. Os proprietários assinaram um termo de autorização de participação dos animais na pesquisa, antes da realização da mesma.

\section{Protocolo experimental}

Os animais foram submetidos a jejum hídrico de seis horas e jejum alimentar de 12 horas, previamente ao exame radiográfico. Na manhã do experimento, cada animal foi pesado e, após a mensuração dos valores basais dos parâmetros a serem analisados, foram sedados com xilazina ${ }^{6}$, na dose de $1 \mathrm{mg} / \mathrm{kg}$ por via intramuscular (IM).

\section{Grupos experimentais}

Os animais foram distribuídos de forma aleatória e equitativa em dois grupos experimentais, cada um possuindo cinco animais. No Grupo 1 (G1) foi administrado o iohexol ${ }^{7}$, na dose de $0,22 \mathrm{ml} / \mathrm{kg}$, e no

\footnotetext{
${ }^{6}$ Dopalen - VetBrands Divisão Veterinária Ltda.

${ }^{7}$ Omnipaque 180 mgI/ml - GE Healthcare Inc.
}

Grupo 2 (G2) foi administrado iohexol, na dose de $0,3 \mathrm{ml} / \mathrm{kg}$. Em ambos os grupos a administração do iohexol foi realizada pela via epidural lombossacra, 15 minutos após a administração da xilazina.

Todas as punções epidurais foram realizadas pela mesma pessoa, todos os animais foram posicionados em decúbito esternal seguindo a técnica citada por Futema (2009b). O local de punção foi o lombossacro, empregando-se uma agulha hipodérmica $30 \times 8$, após tricotomia, assepsia local rigorosa e realização de um botão anestésico com $0,5 \mathrm{ml}$ de lidocaína $2 \%$ com vasoconstrictor ${ }^{8}$. O bisel da agulha foi direcionado cranialmente, durante a administração do meio de contraste, que teve duração padronizada em dois a três minutos. A punção correta do espaço epidural foi confirmada pela aspiração do contraste previamente depositado no canhão da agulha hipodérmica ou, nos casos em que não ocorreu tal aspiração, pela ausência de resistência à administração do contraste. Após a deposição do iohexol, os animais foram mantidos em decúbito esterno abdominal e após quatro minutos foram encaminhados para a sala de radiografia, onde foram radiografados.

O exame radiográfico foi realizado cinco minutos após a administração do meio de contraste. Para este exame foi empregado um aparelho de raios-X de $100 \mathrm{~mA}$ e $100 \mathrm{KV}$ de potência ${ }^{9}$ e filmes radiográficos tamanho $30 \times 40$ centímetros $^{10}$, com o animal contido em decúbito lateral direito.

\section{Avaliação paramétrica}

Foram avaliadas frequência cardíaca, respiratória, temperatura corpórea e eletrocardiografia. A eletrocardiografia (duração da onda P, intervalo P-R, complexo QRS e intervalo Q-T) foram avaliados com o eletrocardiógrafo computadorizado ${ }^{11}$, com

\footnotetext{
${ }^{8}$ Anestésico Bravet - Laboratórios Bravet Ltda.

${ }^{9}$ Aparelho de Raios-X Roentrax monobloco, modelo 100/100 - EMIC - Eletromedicina Ind. Com. Ltda.

${ }^{10}$ Filme radiográfico 30x40 - Kodak Brasileira Ind. Com. Ltda.

${ }^{11}$ Eletrocardiógrafo TEB ECGPC - Tecnologia Eletrônica Brasileira Ltda.
} 
agulhas de metal inseridas subcutaneamente nas regiões umeral e femoral distais de ambos os lados, com o animal contido em decúbito lateral direito. A derivação-padrão para as mensurações foi a DII.

Todos os parâmetros foram mensurados imediatamente antes da administração da xilazina (T-15) e da administração do iohexol (T0) e aos 10 (T10), 20 (T20), 30 (T30), 40 (T40), 50 (T50) e 60 (T60) minutos após esta.

\section{Avaliação não-paramétrica}

A migração cranial do meio de contraste foi determinada em cada animal a partir da radiografia, determinando-se qual a vértebra mais cranial cujo espaço epidural apresentou toda sua extensão opacificada pelo contraste e a distância em centímetros percorridos pelo mesmo, a partir do ponto de administração.

A avaliação da migração do meio de contraste de todos os animais foi realizada pelo mesmo indivíduo, o qual não teve conhecimento de qual dose de meio de contraste foi empregada (estudo cego).

Após o período experimental, os animais foram liberados para retornarem às suas residências.

\section{Análise estatística}

Foi realizada empregando o programa Graphpad Instat. Os dados paramétricos foram analisados com o emprego da análise de variância para amostras repetidas e a comparação entre os momentos e entre os grupos foi realizada pelo teste de StudentNewman-Keuls. Os dados referentes à migração do contraste foram avaliados empregando o teste $t$ de Student. Todos os testes foram aplicados ao nível de $5 \%$ de significância.

Os dados estão apresentados, nas tabelas e no texto, na forma de média \pm desvio padrão.

\section{Resultados e Discussão}

A punção do espaço epidural foi facilmente conseguida e em nove dos animais pôde ser confirmada pela observação da aspiração do meio de contraste previamente depositado no canhão da agulha, conforme relatado por Muir III et al. (2001) e por Massone (2011). No animal em que não se observou a aspiração do meio de contraste, a localização correta da agulha foi feita pela ausência de resistência ao êmbolo da seringa.

A altura máxima individual atingida pelo meio de contraste foi até a $13^{\mathrm{a}}$ vértebra torácica o Grupo 1 e até a $12^{\mathrm{a}}$ vértebra torácica no Grupo 2, obtendose como médias a $2^{\mathrm{a}}$ vértebra lombar (Figura 1) e a $1^{\text {a }}$ vértebra lombar (Figura 2), respectivamente. A altura máxima média em centímetros foi significativamente maior no Grupo $2(12,1 \pm 2,9)$ que no Grupo $1(10,2 \pm 1,7)$ (Tabela 1). Estes resultados estão de acordo com os relatados por Johnson et al. (1996), os quais informaram que a dose de contraste de $0,2 \mathrm{~mL} / \mathrm{kg}$ propiciaram a sua migração até a segunda vértebra lombar no qual foram obtidos resultados semelhantes no presente trabalho.

Figura 1.Radiografia de um animal do Grupo 1 demonstrando migração do contraste até a $2^{\mathrm{a}}$ vértebra lombar (seta).

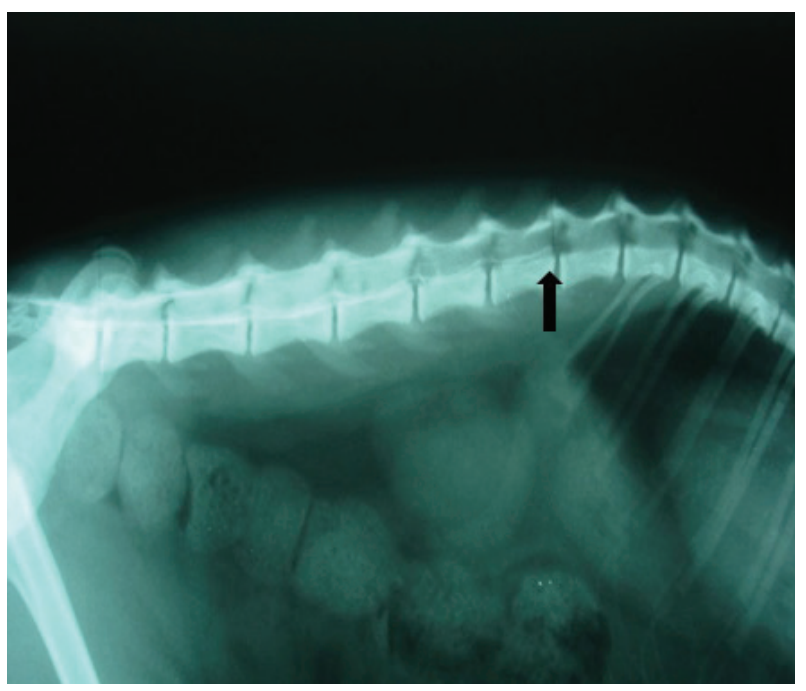

Fonte: Elaboração dos autores. 
Figura 2.Radiografia de um animal do Grupo 2 demonstrando migração do contraste até a $1^{\mathrm{a}}$ vértebra lombar (seta).

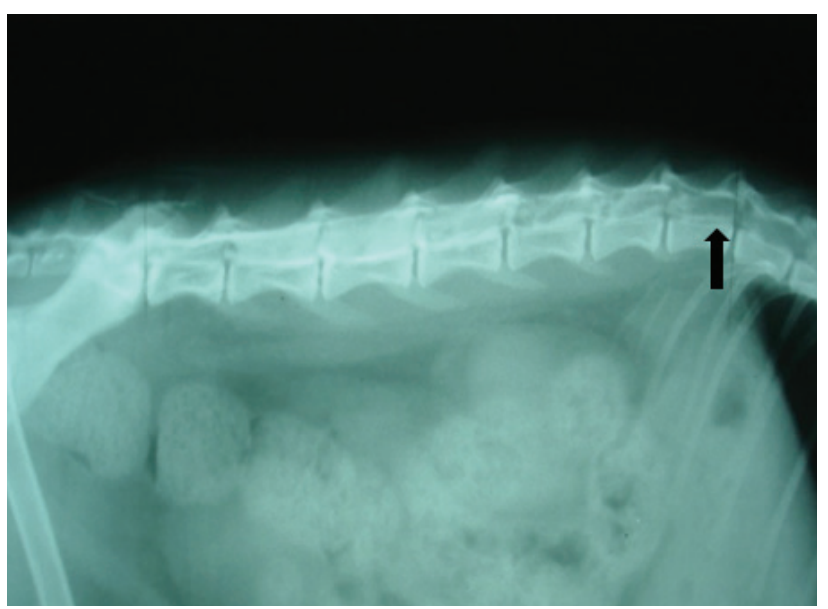

Fonte: Elaboração dos autores.

Tabela1. Altura máxima atingida pelo meio de contraste (em localização anatômica e em centímetros) decorrente da administração epidural de iohexol, nas doses de $0,22 \mathrm{~mL} / \mathrm{kg}$ (Grupo 1) e 0,3 mL/kg (Grupo 2), em felinos.

\begin{tabular}{|c|c|c|c|c|c|}
\hline \multicolumn{3}{|c|}{ Grupo 1} & \multicolumn{3}{|c|}{ Grupo 2} \\
\hline \multirow{2}{*}{ Animal } & \multicolumn{2}{|c|}{ Altura máxima } & \multirow{2}{*}{ Animal } & \multicolumn{2}{|c|}{ Altura máxima } \\
\hline & Vértebra & $\mathrm{cm}$ & & Vértebra & $\mathrm{cm}$ \\
\hline 02 & L2* & 9,3 & 01 & $\mathrm{~L}^{\#}{ }^{\#}$ & 8 \\
\hline 05 & L2 & 10,6 & 03 & L1 & 13 \\
\hline 06 & T13\# & 13 & 04 & $\mathrm{~T} 12$ & 16 \\
\hline 07 & L4 & 8,5 & 08 & L3 & 11,7 \\
\hline 09 & L3 & 9,5 & 10 & $\mathrm{~L} 1$ & 12 \\
\hline Média $\pm \mathrm{DP} * *$ & $\mathrm{~L} 2$ & $10,2 \pm 1,7$ & & L1 & $12,1 \pm 2,9$ \\
\hline
\end{tabular}

*L - Vértebra Lombar **DP - Desvio Padrão

\# T- Vértebra torácica

Fonte: Elaboração dos autores.

A maior migração cranial do meio de contraste obtida com a dose de $0,3 \mathrm{~mL} / \mathrm{kg}$ comprovou as citações de Skarda e Muir III (1996) e Valadão et al. (1990), a respeito da importância desta variável na dispersão cranial do anestésico local administrado por esta via. Provavelmente, a injeção de um anestésico local nesta dose, pela mesma via aqui empregada, propiciaria a anestesia completa dos pedículos ovarianos de felinos, já que os nervos espinhais L3 e L4 seriam bloqueados (BAILEY et al., 1988).

A administração epidural do iohexol comprovou ser um procedimento que de maneira segura e prática comprova a importância da variação do volume administrado na migração cranial do mesmo, conforme relataram Curatolo et al. (1994), Lopez et al. (1997), Hendrickson et al. (1998), Vas et al. (2003), Lee et al. (2004, 2005), Landsdowne et al. (2005), Gorgi et al. (2006).

A administração do iohexol não interferiu significativamente na frequência cardíaca (Tabela 2 e Figura 3) dos animais. Os valores de frequência cardíaca se mantiveram dentro dos parâmetros fisiológicos normais para a espécie felina 
(CAMACHO; MUCHA, 2004) durante todo o estudo. Isto comprova a segurança da epidurografia com o iohexol nesta espécie animal, quanto a este parâmetro.

Tabela 2. Variação (média \pm desvio padrão) da freqüência cardíaca (batimentos/minuto), em diferentes momentos, de felinos sedados com xilazina e medicados pela via epidural com iohexol, nas doses de 0,22 mL/ $\mathrm{kg}$ (Grupo 1) e 0,3 $\mathrm{mL} / \mathrm{kg}$ (Grupo 2).

\begin{tabular}{ccccccccc}
\hline \multirow{2}{*}{ Grupo } & \multicolumn{7}{c}{ Momento } \\
\cline { 2 - 8 } & T-15 & T0 & T10 & T20 & T30 & T40 & T50 & T60 \\
\hline 1 & $150 \pm 48,9$ & $139 \pm 29,5$ & $134 \pm 28$ & $118 \pm 38,9$ & $133 \pm 23,9$ & $123 \pm 39,6$ & $130 \pm 29,6$ & $126 \pm 23,7$ \\
2 & $159 \pm 19,3$ & $152 \pm 25,9$ & $144 \pm 44,4$ & $149 \pm 44$ & $150 \pm 33,8$ & $139 \pm 34,6$ & $126 \pm 26$ & $141 \pm 38,2$ \\
\hline
\end{tabular}

Fonte: Elaboração dos autores.

No presente trabalho não foi detectada nenhuma alteração decorrente do emprego do iohexol sobre as variáveis eletrocardiográficas estudadas, o que novamente comprova a inocuidade deste meio de contraste sobre a eletrofisiologia cardíaca.

Figura 3. Variação dos valores médios da freqüência cardíaca (batimentos/minuto) em diferentes momentos, de felinos sedados com xilazina e medicados pela via epidural com iohexol, nas doses de $0,22 \mathrm{~mL} / \mathrm{kg}$ (Grupo 1) e 0,3 $\mathrm{mL} / \mathrm{kg}$ (Grupo 2).

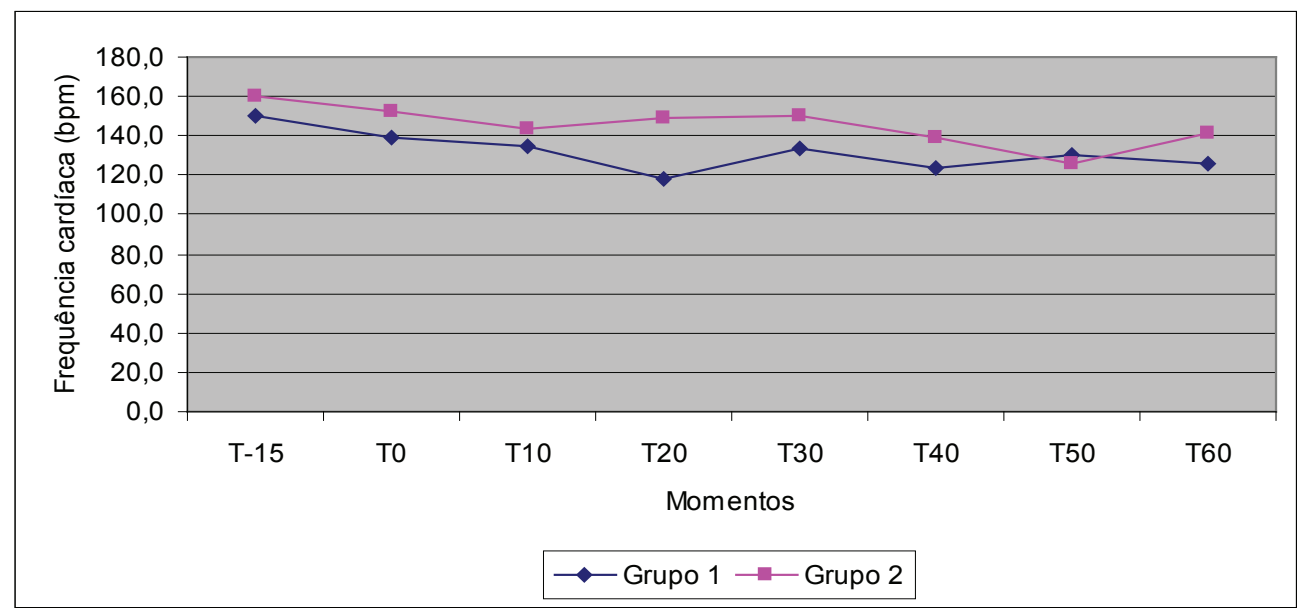

Fonte: Elaboração dos autores.

A duração da onda P representa a despolarização atrial (SEVERIN, 1992) e o tempo normal para esse intervalo em felinos é de 40 milisegundos (CAMACHO; MUCHA, 2004). No entanto, não podemos afirmar que os valores médios de 47 a 55 milisegundos observados nos animais deste experimento (Tabela 3 e Figura 4) sejam anormais para a espécie, já que mesmo antes da administração de qualquer fármaco tais valores já se encontravam elevados. Independentemente disto, observou-se que as administrações da xilazina e a epidurografia não interferiram com a despolarização atrial. 
Tabela 3. Variação (média \pm desvio padrão) da duração da onda $\mathrm{P}(\mathrm{ms})$, em diferentes momentos, de felinos sedados com xilazina e medicados pela via epidural com iohexol, nas doses de $0,22 \mathrm{~mL} / \mathrm{kg}$ (Grupo 1) e $0,3 \mathrm{~mL} / \mathrm{kg}$ (Grupo 2).

\begin{tabular}{ccccccccc}
\hline \multirow{2}{*}{ Grupo } & \multicolumn{7}{c}{ Momento } \\
\cline { 2 - 9 } & T-15 & T0 & T10 & T20 & T30 & T40 & T50 & T60 \\
\hline 1 & $48 \pm 3,7$ & $51 \pm 7,2$ & $47 \pm 3,8$ & $51 \pm 4,3$ & $55 \pm 8,4$ & $53 \pm 5,4$ & $49 \pm 6,2$ & $49 \pm 6,4$ \\
2 & $48 \pm 8,6$ & $51 \pm 6,2$ & $50 \pm 8,6$ & $50 \pm 6,3$ & $50 \pm 8,2$ & $49 \pm 7,1$ & $49 \pm 7,8$ & $48 \pm 12,4$ \\
\hline
\end{tabular}

Fonte: Elaboração dos autores.

Figura 4. Variação dos valores médios da duração da onda $\mathrm{P}(\mathrm{ms})$, em diferentes momentos, de felinos sedados com xilazina e medicados pela via epidural com iohexol, nas doses de $0,22 \mathrm{~mL} / \mathrm{kg}$ (Grupo 1) e 0,3 mL/kg (Grupo 2).

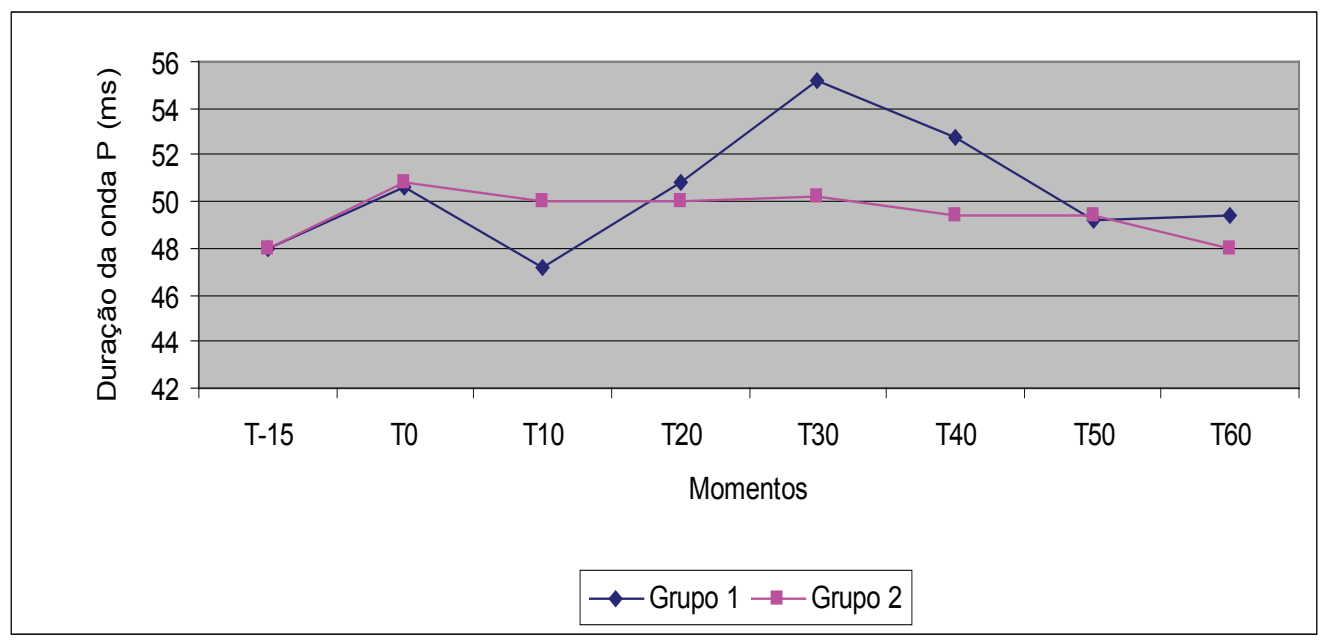

Fonte: Elaboração dos autores.

O intervalo P-R corresponde ao tempo em que o impulso elétrico está despolarizando o nodo átrioventricular e os ramos direito e esquerdo do feixe de His (SEVERIN, 1992). Neste experimento os valores obtidos permaneceram estáveis (Tabela 4 e Figura 5) e dentro dos limites fisiológicos para felinos, durante todo o período experimental, como relatado por Camacho e Mucha (2004).

Tabela 4. Variação (média \pm desvio padrão) do intervalo PR (ms), em diferentes momentos, de felinos sedados com xilazina e medicados pela via epidural com iohexol, nas doses de $0,22 \mathrm{~mL} / \mathrm{kg}$ (Grupo 1) e $0,3 \mathrm{~mL} / \mathrm{kg}$ (Grupo 2).

\begin{tabular}{rcccccccc}
\hline \multirow{2}{*}{ Grupo } & \multicolumn{7}{c}{ Momento } \\
\cline { 2 - 8 } & T-15 & T0 & T10 & T20 & T30 & T40 & T50 & T60 \\
\hline 1 & $63 \pm 7,9$ & $69 \pm 11,4$ & $67 \pm 8,3$ & $80 \pm 13,6$ & $78 \pm 16,9$ & $77 \pm 18,1$ & $72 \pm 13,3$ & $75 \pm 16,6$ \\
2 & $65 \pm 11,8$ & $71 \pm 14,6$ & $67 \pm 13,8$ & $69 \pm 15,3$ & $69 \pm 14,9$ & $73 \pm 13,9$ & $75 \pm 12,7$ & $71 \pm 18,3$ \\
\hline
\end{tabular}

Fonte: Elaboração dos autores. 
Figura 5. Variação dos valores médios do intervalo entre as ondas $P$ e $R(\mathrm{~ms})$, em diferentes momentos, de felinos sedados com xilazina e medicados pela via epidural com iohexol, nas doses de $0,22 \mathrm{~mL} / \mathrm{kg}$ (Grupo 1) e $0,3 \mathrm{~mL} / \mathrm{kg}$ (Grupo 2).

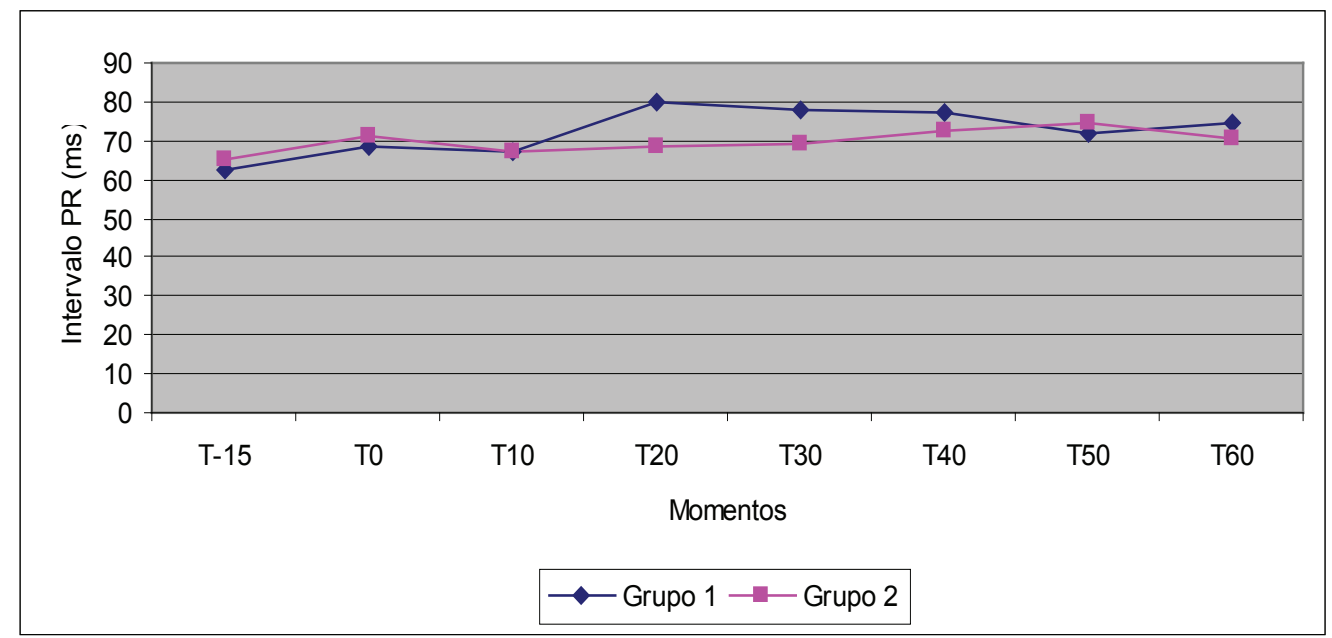

Fonte: Elaboração dos autores.

O complexo QRS compreende o período de os valores obtidos (Tabela 5 e Figura 6) com a despolarização ativa da musculatura ventricular administração epidural de iohexol não interfere (SEVERIN, 1992) e Camacho e Mucha (2004) com a despolarização ventricular, uma vez que os consideram como normais para felinos valores valores da duração do QRS já estavam elevados abaixo de 60 milisegundos. Pode-se deduzir, que antes da administração de quaisquer fármacos.

Tabela 5. Variação (média \pm desvio padrão) da duração do complexo QRS (ms), em diferentes momentos, felinos sedados com xilazina e medicados pela via epidural com iohexol, nas doses de $0,22 \mathrm{~mL} / \mathrm{kg}$ (Grupo 1) e $0,3 \mathrm{~mL} / \mathrm{kg}$ (Grupo 2).

\begin{tabular}{ccccccccc}
\hline \multirow{2}{*}{ Grupo } & \multicolumn{7}{c}{ Momento } \\
\cline { 2 - 8 } & T-15 & T0 & T10 & T20 & T30 & T40 & T50 & T60 \\
\hline 1 & $74 \pm 9,2$ & $71 \pm 9,6$ & $71 \pm 3,8$ & $71 \pm 7,6$ & $72 \pm 9,5$ & $69 \pm 6,1$ & $66 \pm 6,3$ & $64 \pm 8,6$ \\
2 & $69 \pm 8,9$ & $75 \pm 9,0$ & $75 \pm 9,2$ & $78 \pm 10,3$ & $75 \pm 7,4$ & $80 \pm 8,3$ & $73 \pm 4,8$ & $76 \pm 4,4$ \\
\hline
\end{tabular}

Fonte: Elaboração dos autores. 
Figura 6. Variação dos valores médios da duração do complexo QRS (ms), em diferentes momentos, de felinos sedados com xilazina e medicados pela via epidural com iohexol, nas doses de $0,22 \mathrm{~mL} / \mathrm{kg}$ (Grupo 1) e $0,3 \mathrm{~mL} / \mathrm{kg}$ (Grupo 2).

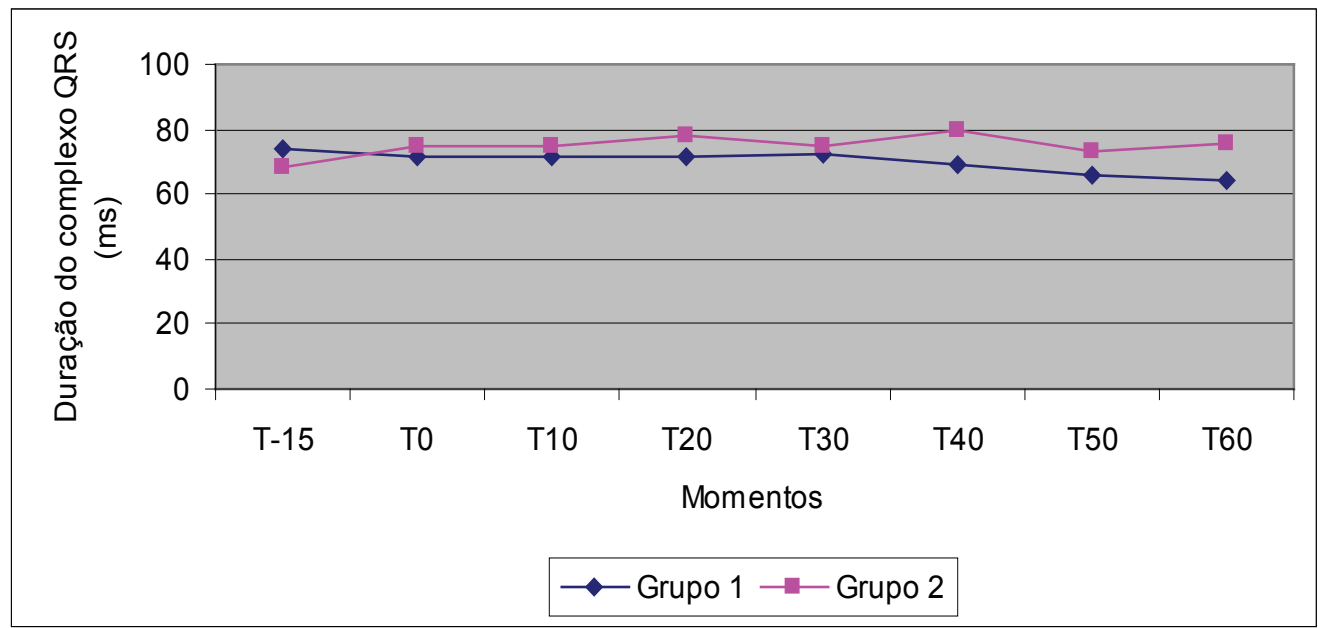

Fonte: Elaboração dos autores.

O intervalo Q-T representa a sístole 7) este parâmetro se manteve dentro dos padrões ventricular do coração, ou seja, a despolarização normais para a raça como relata Camacho e Mucha e a repolarização dos ventrículos, e varia de modo (2004), confirmando mais uma vez a segurança da inverso à freqüência cardíaca (SEVERIN, 1992). epidurografia com iohexol sobre a eletrofisiologia Durante todo o experimento (Tabela 6 e Figura cardíaca, em felinos.

Tabela 6. Variação (média \pm desvio padrão) do intervalo QT (ms), em diferentes momentos, de felinos sedados com xilazina e medicados pela via epidural com iohexol, nas doses de $0,22 \mathrm{~mL} / \mathrm{kg}$ (Grupo 1) e $0,3 \mathrm{~mL} / \mathrm{kg}$ (Grupo 2).

\begin{tabular}{ccccccccc}
\hline \multirow{2}{*}{ Grupo } & \multicolumn{7}{c}{ Momento } \\
\cline { 2 - 9 } & T-15 & T0 & T10 & T20 & T30 & T40 & T50 & T60 \\
\hline 1 & $175 \pm 31,1$ & $203 \pm 34,3$ & $209 \pm 47,4$ & $210 \pm 34,2$ & $216 \pm 36$ & $200 \pm 26,9$ & $209 \pm 27,1$ & $202 \pm 24$ \\
2 & $173 \pm 28,7$ & $189 \pm 26,3$ & $186 \pm 37,6$ & $189 \pm 39,8$ & $192 \pm 40,8$ & $193 \pm 35,8$ & $202 \pm 29,4$ & $198 \pm 34,6$ \\
\hline
\end{tabular}

Fonte: Elaboração dos autores. 
Figura 7. Variação dos valores médios do intervalo entre as ondas Q e $\mathrm{T}(\mathrm{ms})$, em diferentes momentos, de felinos sedados com xilazina e medicados pela via epidural com iohexol, nas doses de $0,22 \mathrm{~mL} / \mathrm{kg}$ (Grupo 1) e $0,3 \mathrm{~mL} / \mathrm{kg}$ (Grupo 2).

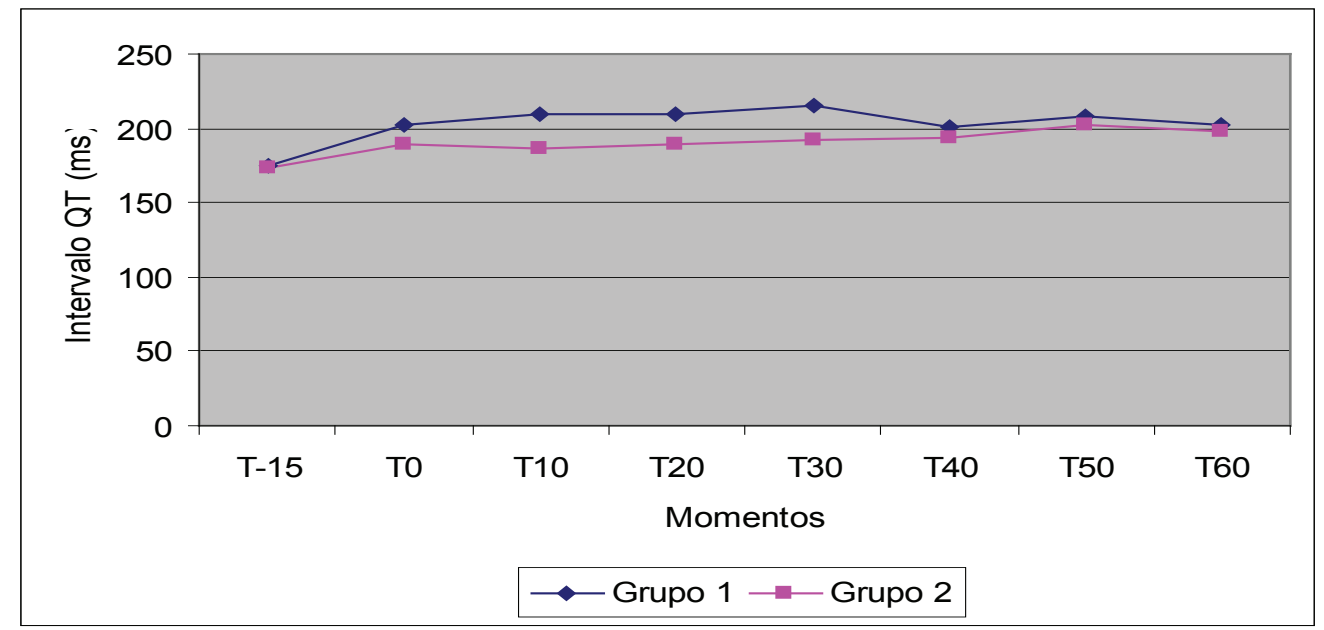

Fonte: Elaboração dos autores.

Os valores obtidos para a frequência respiratória (Tabela 7 e Figura 8) já se encontravam acima dos limites fisiológicos para felinos (CAMACHO; MUCHA, 2004) no momento T-15. Este achado pode estar relacionado à excitação que os animais apresentaram no início do período experimental e especialmente à contenção inicial para mensuração dos parâmetros fisiológicos.

Tabela 7. Variação (média \pm desvio padrão) da frequência respiratória (movimentos/minuto), em diferentes momentos, de felinos sedados com xilazina e medicados pela via epidural com iohexol, nas doses de $0,22 \mathrm{~mL} / \mathrm{kg}$ (Grupo 1) e 0,3 $\mathrm{mL} / \mathrm{kg}$ (Grupo 2).

\begin{tabular}{rcccccccc}
\hline \multirow{2}{*}{ Grupo } & \multicolumn{7}{c}{ Momento } \\
\cline { 2 - 8 } & T-15 & T0 & T10 & T20 & T30 & T40 & T50 & T60 \\
\hline 1 & $56 \pm 19,4$ & $44 \pm 11$ & $46 \pm 17,8$ & $45 \pm 11,8$ & $46 \pm 14,4$ & $48 \pm 11,8$ & $43 \pm 11,9$ & $46 \pm 15$ \\
2 & $56 \pm 8,1$ & $54 \pm 14,6$ & $56 \pm 20,3$ & $67 \pm 38$ & $67 \pm 42,2$ & $70 \pm 45,5$ & $63 \pm 37,5$ & $67 \pm 40,5$ \\
\hline
\end{tabular}

Fonte: Elaboração dos autores. 
Figura 8. Variação dos valores médios da frequência respiratória (movimentos/minuto), em diferentes momentos, de felinos sedados com xilazina e medicados pela via epidural com iohexol, nas doses de $0,22 \mathrm{~mL} / \mathrm{kg}$ (Grupo 1) e 0,3 $\mathrm{mL} / \mathrm{kg}$ (Grupo 2).

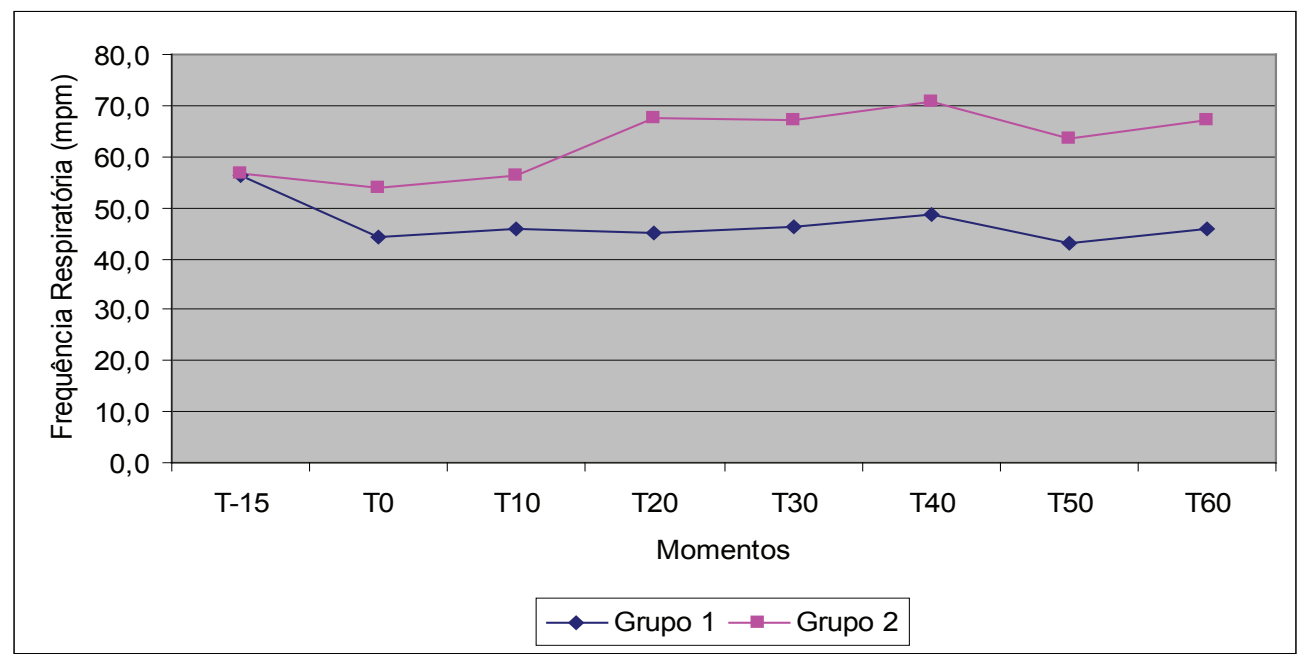

Fonte: Elaboração dos autores.

Houve redução estatisticamente significativa da temperatura corpórea no Grupo 1 a partir do momento T40. No momento T60, a temperatura média no Grupo 1 foi menor que a do Grupo 2 (Tabela 8 e Figura 9).

Tabela 8. Variação (média \pm desvio padrão) da temperatura corpórea $\left({ }^{\circ} \mathrm{C}\right)$, em diferentes momentos, de felinos sedados com xilazina e medicados pela via epidural com iohexol, nas doses de $0,22 \mathrm{~mL} / \mathrm{kg}$ (Grupo 1) e $0,3 \mathrm{~mL} / \mathrm{kg}$ (Grupo 2).

\begin{tabular}{ccccccccc}
\hline \multirow{2}{*}{ Grupo } & \multicolumn{7}{c}{ Momento } \\
\cline { 2 - 9 } & T-15 & T0 & T10 & T20 & T30 & T40 & T50 & T60 \\
\hline 1 & $38,1 \pm 0,5$ & $37,8 \pm 0,7$ & $37,7 \pm 0,6$ & $37,4 \pm 0,8$ & $37,3 \pm 0,6$ & $37 \pm 0,8^{*}$ & $36,9 \pm 0,8^{*}$ & $36,5 \pm 0,9^{*}$ \\
2 & $38,1 \pm 0,7$ & $38,4 \pm 0,4$ & $38,3 \pm 0,4$ & $38,1 \pm 0,8$ & $38 \pm 0,8$ & $37,9 \pm 1,2$ & $37,9 \pm 1,2$ & $38 \pm 1,1^{* *}$ \\
\hline
\end{tabular}

*Estatisticamente diferente do valor inicial (T-15).

**Estatisticamente diferente do Grupo 1.

Fonte: Elaboração dos autores. 
Figura 9. Variação dos valores médios da temperatura corpórea $\left({ }^{\circ} \mathrm{C}\right)$, em diferentes momentos, de felinos sedados com xilazina e medicados pela via epidural com iohexol, nas doses de $0,22 \mathrm{~mL} / \mathrm{kg}$ (Grupo 1) e $0,3 \mathrm{~mL} / \mathrm{kg}$ (Grupo 2).

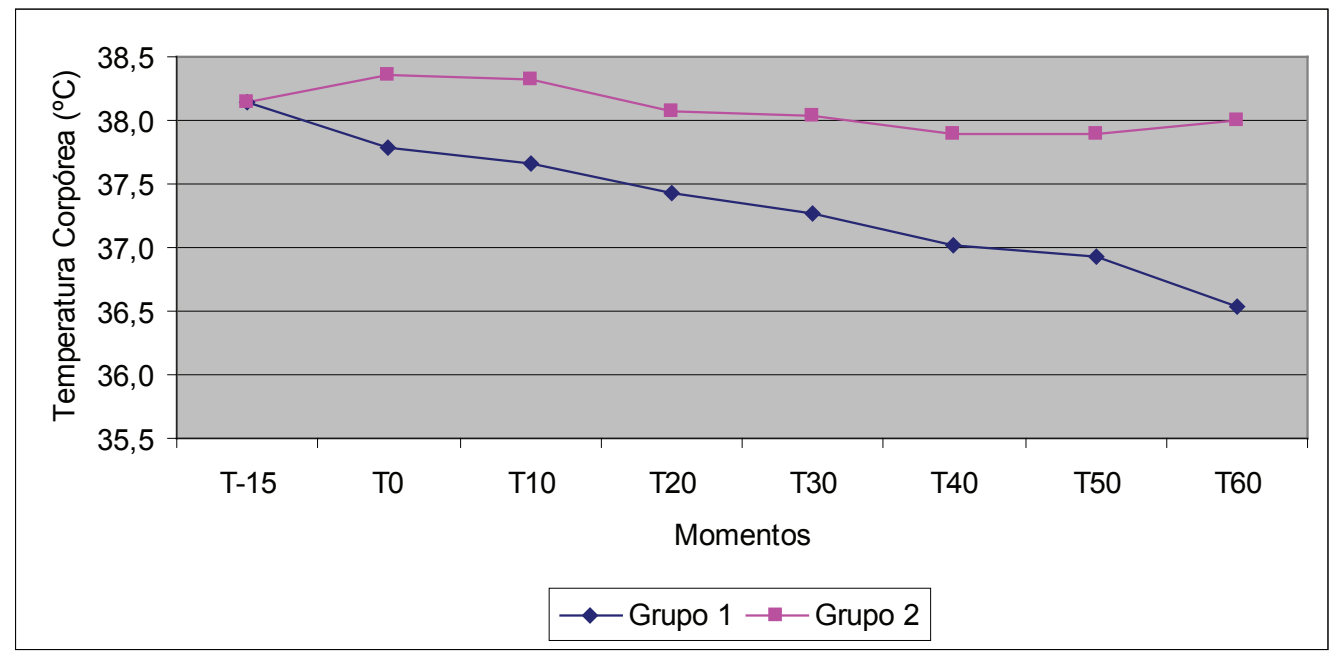

Fonte: Elaboração dos autores.

A temperatura média da sala onde foi realizado o experimento foi mantida estável em $25^{\circ} \mathrm{C}$ para todos os animais. No entanto foi registrada hipotermia nos animais do Grupo 1, considerando os valores normais para a espécie de $37,8^{\circ} \mathrm{Ca} 39,2^{\circ} \mathrm{C}$ citados por Futema (2009a). Este achado provavelmente deveuse ao efeito depressor da xilazina sobre o centro termorregulatório bem como à reduzida atividade muscular apresentada pelos animais, enquanto sedados pelo fármaco (VIRTANEN, 1989). O fato de não ter sido observada hipotermia nos animais do Grupo 2 pode dever-se a uma menor sensibilidade dos mesmos aos efeitos hiportermizantes da xilazina, uma vez que um efeito do iohexol sobre este parâmetro é muito pouco provável.

Pode-se concluir que a administração de iohexol pela via epidural lombossacra, na dose $0,22 \mathrm{~mL} /$ $\mathrm{Kg}$ e $0,3 \mathrm{~mL} / \mathrm{Kg}$, proporcionou migração cranial até a $2^{\circ}$ e $1^{\circ}$ vértebras lombares, respectivamente, e não interferiu significativamente nos parâmetros cardiorrespiratórios e na temperatura corporal dos felinos.

\section{Aspectos Éticos e de Biossegurança}

Todos os procedimentos experimentais foram aprovados pelo Comitê de Ética em Pesquisa (CEP), sob protocolo de número 123/ 2009.

\section{Referências}

BAILEY, C.; KITCHELL, R. L.; HAGHIGHI, S. S.; JOHNSON, R. D. Spinal nerve root origins of the cutaneous nerves of the canine pelvic limb. American Journal of Veterinary Research. Schaumburg, v. 49, n. 1, p. 115-119, 1988.

BRUNSON, D. B. Use of halothane and isoflurane in the horse. The Veterinary Clinics of North America - Equine Practice, Amsterdam, v. 6, n. 3, p. 529-541, 1990.

CAMACHO, A. A.; MUCHA, C. J. Semiologia do sistema circulatório de cães e gatos. In: FEITOSA, F. L. Semiologia veterinária: a arte do diagnóstico. São Paulo: Roca, 2004. p. 282-311.

CURATOLO, M.; ORLANDO, A.; ZBINDEN, A. M.; SCARAMOZZINO, P.; VENUTI, F. S. A multifactorial analysis of the spread of epidural analgesia. Acta Anaesthesiologica Scandinavica, New York, v. 38, n. 7, p. 646-52, 1994.

FUTEMA, F. Avaliação pré-anestésica, In: FANTONI, D. T.; CORTOPASSI, S. R. G. Anestesia em cães e gatos. 2. ed. São Paulo: Roca, 2009a. p. 73-82. 
.Técnicas de anestesia local. In: FANTONI, D. T.; CORTOPASSI, S. R. G. Anestesia em cães e gatos. 2. ed. São Paulo: Roca, 2009b. p. 310-332.

GORGI, A. A.; HOFMEISTER, E. H.; HIGGINBOTHAM, M. J.; KENT, M. Effect of body position on cranial migration of epidurally injected methylene blue in recumbent dogs. American Journal of Veterinary Research, Schaumburg, v. 67, n. 2, p. 219-21, 2006.

HENDRICKSON, D. A.; SOUTHWOOD, L. L.; LOPEZ, M. J.; JOHNSON, R.; KRUSE-ELLIOT, K. T. Cranial migration of different volumes of New-Methylene Blue after caudal epidural injection in the horse. Equine Practice, Amsterdam, v. 20, n. 3, p. 12-4, 1998.

JOHNSON, R. A.; LOPEZ, M. J.; HENDRICKSON, D. A.; KRUSE-ELLIOTT, K. T. Cephalad distribution of three differing volumes of new methylene blue injected into the epidural space in adult goats. Veterinary Surgery, Chicago, v. 25, n. 5, p. 448-451, 1996.

LANDSDOWNE, J. L.; KERR, C. L.; BOURE, L. P.; PEARCE, S. G.Epidural migration of new methylene blue in $0.9 \%$ sodium chloride solution or $2 \%$ mepivacaine solution following injection into the first intercoccygeal space in foal cadavers and anesthetized foals undergoing laparoscopy. American Journal of Veterinary Research, Schaumburg, v. 66, n. 8, p. 1324-9, 2005.

LEE, I.; YAMAGISHI, N.; OBOSHI, K.; AYUKAWA, Y.; SASAKI, N.; YAMADA, H. Distribution of new methylene blue injected into the caudal epidural space in cattle. Veterinary Journal, Amsterdam, v. 169, n. 3, p. 257-61, 2005.

LEE, I.; YAMAGISHI, N.; OBOSHI, K.; YAMADA, $H$. Distribution of new methylene blue injected into the lumbosacral epidural space in cats. Veterinary Anaesthesia Analgesia, New York, v. 31, n. 3, p. 190194, 2004.

LEWIS, D. D.; HOSGOOD, G. Complications associated with the use of iohexol for myelography of the cervical vertebral column in dorgs: 66 cases (1988-1990). Journal American Veterinary Medical Association, Schaumburg, v. 200, n. 9, p. 1381-1384, 1992.
LOPEZ, M. J.; JOHNSON, R.; HENDRICKSON, D. A.; KRUSE-ELLIOTT, K. T. Cranial migration of differing doses of New-Methylene Blue injected into the epidural space after death of calves and juvenile pigs. American Journal of Veterinary Research, Schaumburg, v. 58, n. 3, p. 786-90, 1997.

MASSONE, F. Técnicas anestésicas em cães. In: Anestesiologia veterinária: farmacologia e técnicas. 6 . ed. Rio de Janeiro: Guanabara Koogan, 2011. p. 105-116.

MUIR III, W. W.; HUBBEL, J. A.E.; SKARDA, R.; BEDNARSKI, R. M. Anestesia localem cães e gatos. In:_ Manual de anestesia veterinária. Porto Alegre: Artmed, 2001. p. 89-102.

SEVERIN, G. A. Manual de cardiologia veterinária. Buenos Aires: Hemisfério Sur, 1992. 91 p.

SKARDA, R. T. Local and regional anesthetic techniques: ruminants e swine. In: THURMON, J. C.; TRANQUILI, W. J.; BENSON, G. J. Lumb \& Jones, veterinary anesthesia. Baltimore: Williams \& Wilkins, 1996. p. 479-514.

SKARDA, R. T.; MUIR III, W. W. Analgesic, hemodinamic and respiratory effects of caudal epidurally administered xylazine hydrochloride in mares. American Journal of Veterinary Research, Schaumburg, v. 57, n. 2, p. 193-200, 1996.

VALADÃO, C. A. A.; PINTO, M. R. A.; MARQUES, J. A.; NUNES, N. Analgesia epidural com xilazina. Avaliação cirúrgica e hemogasométrica. Ars Veterinária, Jaboticabal, v. 6, n. 6, p. 125-35, 1990.

VAS, L.; KULKARNI, V.; MALI, M.; BAGRY, H. Spread of radiopaque dye in the epidural space in infants. Paediatric Anaesthesia, San Francisco, v. 13, n. 3, p. 233-43, 2003.

VIRTANEN, R. Pharmacological profiles of medetomidine and its antagonist, atipamezole. Acta Veterinaria Scandinavica Supplementum, London, v. 85, n. 6, p. 29-37, 1989.

WOOD, A. K.; FARROW, B. R.; FAIRBURM, A. J. Cervical myelography in dogs using iohexol. Acta Radiologica Diagnosis, Saint Louis, v. 26, n. 6, p. 76770, 1995. 
